\title{
LES SOUS-PRODUITS AGRO-INDUSTRIELS DISPONIBLES AU MALI PERSPECTIVES DE LEUR UTILISATION DANS LES PRODUCTIONS ANIMALES
}

\author{
N'GOLO TRAORE
}

\begin{abstract}
RESUME
Le Mali importe beaucoup de produits laitiers alors qu'il exporte des aliments du bétail. De la paille de riz est disponible en grande quantité à l'Office du Niger. Les bouts blancs de canne et les fanes d'arachides sont aussi de bons fourrages. La Les bouts blancs de canne et les fanes d'arachides sont aussi de bons fourrages. La
production d'issues de riz s'accroit, celles de graines et de tourteau de coton production d'issues de riz s'accroît, celles de graines et de tourteau de coton
également, alors que les coques sont brû́es. La production de mélasse de 2000 tonnes doit passer à 8000 tonnes. Les sous-produits d'abattoir, de la pêche demeurent peu importants. Les expériences montrent que ces aliments du bétail peuvent être employés à une embouche rentable des bovins.
\end{abstract}

\section{SUMMARY}

Agro industrial by products in Mali. Project for use in animal production

Much of dairy products are imported in Mali while feedstuffs are exported. Large amounts of rice straw are avallable at Niger Office. Sugar cane tops and peanut leaves are also good feedstuffs. The production of rice by products and cotton seed and cake increase. The cotton shells are burned. From 2000 tons, the molasses production must increase to 8000 tons. Slaughter an fisheries by products remains low. Experiments shows the possibility to use feedstuffs for cattle fattening with benefits.

\section{INTRODUCTION}

L'agriculture malienne a été caractérisée au cours des dix dernières années par le développement des cultures industrielles (coton, arachide, canne à sucre) et la création d'industries de transformation (usine d'égrenage de coton, huilerie, sucrerie, rizerie, abattoir frigorifique et conserverie de poissons). Ces différentes industries fournissent des quantités importantes de sous-produits utilisables dans l'alimentation du bétail et dont le stock est appelé à s'accroître avec la naissance de nouveaux complexes agro-industriels.

L'utilisation dans l'élevage de ces aliments de grande valeur a jusqu'ici été limitée à quelques tonnes dans les centres de recherches zootechniques et dans quelques fermes privées, l'essentiel étant ou exporté (graines de coton, tourteau d'arachide), ou insuffisamment exploité (sous-produits de canne).

Nous assistons également au paradoxe commun aux pays en voie de développement et consistant à importer des pays industrialisés des quantités élevées de lait et produits laitiers et à exporter vers les pays développés d'Europe, d'Amérique et d'Asie, des milliers de tonnes de graines de coton, de tourteau d'arachide et de coton. D'après les statistiques de la F.A.O. (1) les pays du tiers monde importent annuellement pour 500 millions de dollars U.S.(1) de lait concentré sucré et non sucré, lait en poudre et de produits laitiers et exportent 7 millions de tonnes de

(1) Revue Mondiale de Zootechnie, $n^{\circ} 1$, 1972. Activités de la F.A.O., par H.-A. Jasiorowski. sous-produits des huileries, 2,5 millions de tonnes de farine de poisson.

En 1972, le Mali a importé 1200 tonnes de lait et produits laitiers pour 405000000 de francs maliens, tandis que nos exportations de graines de coton atteignent 15900 tonnes pour 676026500 francs maliens, de tourteau d'arachide 8000 tonnes pour 416125111 francs maliens, de tourteau de coton 4000 tonnes pour 216000000 de francs maliens.

Mon propos n'est pas d'épiloguer sur les causes de ce paradoxe dont les plus importantes généralement avancées sont :

1) Le caractère extensif ; transhumant de notre élevage et l'absence de structures modernes de produc tion ;

2) La faible productivité du bétail tropical ;

3) Le bas niveau du prix de la viande;

4) Le cours mondial des aliments du bétail favorisant l'exportation vers les pays d'élevage intensif des sous-produits de grande valeur à des prix rémunérateurs pour nos producteurs.

Les sous-produits agro-industriels ont fait l'objet de multiples rapports d'inventaire : MONGODIN (1965), LECLERQ (1970), BINE YALCOUE (1970). Je ne ferais donc que reprendre dans leurs grandes lignes ces différents inventaires, en les actualisant et en essayant de faire des projections sur l'avenir.

Mais je voudrais à la lumière des recherches effectuées ces dernières années sur les possibilités de l'embouche bovine en milieu tropical replacer le débat sur l'utilisation dans nos programmes d'élevage 
des sous-produits de l'agriculture et des industries de transformation dans ce contexte nouveau et analyser le róle de ce disponible fourrager dans la stratégie du développement de nos productions animales.

L'exposé comporte 3 partícs :

1) Inventaire et localisation géographique des sousproduits agro-industriels;

2) Destination actuelle et économie de leur utilisation dans les productions animales;

3) Esquisse d'une stratégie du développement des productions animales intégrant la consommation des aliments jusqu'ici exportés.

\section{I. - INVENTAIRE ET LOCALISATION GEO GRAPHIQUE DES SOUS-PRODUITS AGRO- INDUSTRIELS}

\section{I.1. Les sous-produits de récolte}

La récolte des céréales laisse sur les champs des tiges (mil, sorgho, maïs) et des pailles (riz, fonio) qui constituent pour le bétail une nourriture d'appoint non négligeable en début de saison sèche. Certains de ces déchets de récolte comme la paille de riz peuvent devenir la base d'aliment grossier d'un systeme de production rationnelle de viande.

Nous ne parlerons ici que de ces derniers.

C'est le cas dans les casiers rizicoles de l'Office du Niger, à Niono, Kourouma et Kolongotomo. Ce sera prochainement le cas dans les plaines semi-aménagées de l'opération riz de Sćgou.

I.1.1. Evaluation du stock de paille dans les casiers de l'Office du Niger

A l'Office du Niger la récolte de riz commence en novembre et finit en fin décembre. Les souches donjent des repousses plus ou moins abondantes selon la variété de riz et l'état d'humidité du sol, et qui sont envahies par les animaux venus de la transhumance dès la mise en gerbier des épis qui a lieu deux à trois semaines après la moisson.

Au cours des essais de rendement effectués pax l'I.R.A.T. et la Station du Sahel sur diverses variétés de riz cultivé dans la zone, le rapport: gain/paille a été déterminé et varie entre $21 \mathrm{p} .100$ et 54 p. 100 pour les rendements en grains s'étalant de $1,9 \mathrm{t} / \mathrm{ha}$ à $2,8 \mathrm{t}$ /ha (2). La quantilé de paille prélevée avec les épis peut varier avec les moissonneurs. Pour évaluer le stock de paille nous avons retent un rapport gains/ paille de 25 p. 100 pour les casiers de l'Office du Niger et les plaines semi-aménagées de l'Opération riz de Ségou. Ainsi le stock de paille disponible en 1972-1973 à l'Office du Niger pouvait être estimé à 90000 tonnes.

L'utilisation de la paille de riz dans l'alimentation du bétail pose le problème du condilionnement. Des observations faites à la Station du Sahel ont permis d'établir le prix de revient du kilo de paille bottelée, 4,35 FM rendu à l'étable, soit 12.4 FM l'unité fourragère.

L'évolution des stocks prévisibles au cours des cinq prochaines années est résumée dans un tableau en annexe.

La valeur bromatologique de quelques ćchantillons de paille de riz analysés par le laboratoire de nutrition de l'I.E.M.V.T. est donné en annexe.

La valeur fourragère de la paille de riz est d'environ $0,35 \mathrm{UF} / \mathrm{kg}$. La paille de riz mélassée à $20 \mathrm{p} .100$ a une valeur fourragère de $0,43 \mathrm{UF} / \mathrm{kg}$ (bovin).

1.1.2. Les sous-produits de récolte de la canne à sucre: les bouts blancs de canne

Les bouts blancs de canne sont constitués par l'ex-

(2) Rapport Station du Sahel, 1970. trémité feuillue de la plante sectionnée et laissée dans le champ au moment de la coupe. Les plantaLions de canne de Dougabougou couvrant une superficie de 1300 hectares fournissent la matière première à la sucrerie d'une capacité de 6000 tonnes de sucre raffiné en poudre.

La coupe de canne laisse des quantités très appréciables de bouts blancs de canne. Des enquêtes effecluées par la Station du Sahel et le Secteur de production de Dougabougou ont permis d'estimer les bouts blancs de canne à 15-25 p. 100 du poids total de la canne coupée.

Nous retenons une moyenne de 20 p. 100 pour le calcul du stock fourrager, ce qui donne les prévisions de production résumées dans un tableau on annexe.

En 1969-1970 l'usine a broyé 55000 tounes de canne, ayant donné environ 11000 tonnes de bouts blancs de canne. Malheureusement la méthode de coupe manuelle utilisée ne permet pas de récupérer cet important tonnage de fourrage vert. En effet la canne est brûlée 24 heures avant la coupe pour faciliter les opérations et augmenter la productivité du travail.

Le programme d'extension de la culture de canne prévoit le démarrage à Séribala à $30 \mathrm{~km}$ de Niono d'une deuxième sucrerie en 1975 dont la capacité de production triplera cclle de Dougabougou. A Séribala les parcelles de canne sont installées de façon à permettre une coupe mécanique de la canne, résolvant annsi le probleme de la récupération des bouts blancs dont le disponible peut être estimé à 37000 tonnes à partir de 1975. La valeur bromatologique de ce fourrage d'après les analyses du Laboratoire de nutrition de l'I.E.M.V.T. est de $0,15 \mathrm{UF}$ et $5 \mathrm{~g}$ de MAD par kilo.

Dans certains pays d'Amérique latine producteurs de sucre, les bouts blancs de canne sont bien valorisés dans les productions de viande et de lait.

Les expériences d'encraissement intensif de bovins à la sucrerie de Dougabougou ont été faites avec les bouts blancs de camne comme fourrage de base. Le prix de revient du lourrage qui se ramène aux frais de ramassage et de transport est de $1,25 \mathrm{~F} / \mathrm{kg}$, soit 8 FM par unité fourragère.

\subsubsection{Les fanes d'arachide}

La culture de l'arachide introduite au Mali depuis longtemps connaît depuis 10 ans une extension progressive. Le bond a été surtout important à partir de 1967-1968. L'Opération arachide qui encadre les zones de production $\mathrm{y}$ diffuse des thèmes de vulgarisation aussi bien dans le domaine agricole que dans celui d'une association agriculture-élevage.

Les fanes d'arachide issues de la récolte des gousses constituent un excellent fourrage quand elles sont bien récoltées. Mais lorsque la récolte se fait tard les pertes en feuilles sont nombreuses, ce qui diminue la valeur fourragère. Les quantités de fanes d'arachide récoltćes en 1972 d'après l’Opération ara chide peuvent être estimées ainsi :

$$
\begin{array}{r}
\text { Zone encadrée } \ldots \ldots \ldots \ldots \ldots \ldots, \quad 130000 \text { tomnes } \\
\text { Zone non encadrée } \ldots \ldots \ldots \ldots \ldots, 40000 \text { tonnes } \\
\text { Total } \ldots \ldots \ldots \ldots \ldots \ldots \ldots \ldots, 170000 \text { ionnes }
\end{array}
$$

L'évolution de la production estimée d'après les objectifs de production arachidière est donnée en annexe. La valeur fourragère moyenne est d'environ $0,4 \mathrm{UF} / \mathrm{kg}$ et $6 \mathrm{~g}$ MAD par kilo.

\section{I.2. Les sous-produits d'usinage du paddy}

Le décorticage du paddy se fait à l'office du Niger dans trois rizeries situées respectivement à Molodo, 
Kourouma et Kolongotomo. Une autre usine de décorticage vient de démarrer à Sćvaré (Mopti) et doit usiner le riz produit dans les $\mathbf{p}$ inines semi-aménagées des cooperations riz de Mopti et Ségou. Les capacités théoriques des quatre usines sont :

Molodo ....... 18000 tonnes

Kourouma ..... 18000 tonnes

Kolongotomo .. 12000 tonnes

Sévaré ........ 13000 tomnes de paddy par an

Les principaux sous-produits sont les sons et farines basses de riz.

Les trois rizeries de l'Office du Niger ont produit en 19722808 tonnes de son et farine basse de riz, en 1972-73 dont 1104 tonnes de son, 1704 tonnes de farine basse.

D'après la division commerciale de l'Office du Niger, le son représente 2,3 p. 100 et la farine basse 3,5 p. 100 du paddy usiné.

Les analyses bromatologiques effectuées sur plusieurs échantillons par l'I.E.M.V.T. donnent les résultats suivants (bovins)

Son de riz: $0,32 \mathrm{UF} / \mathrm{kg}$ el $31 \mathrm{~g}$ de MAD/kg; kg.

Farine basse de riz : $1,10 \mathrm{UF} / \mathrm{kg}$ et $57-70 \mathrm{~g}$ de MAD/

L'usine de Sévaré qui décortique du riz étuvé a produit 300 tonnes de son et farine basse de riz en 1972-73.

Le prix de cession de ces sous-produits carreau usine est de $5 \mathrm{~F}$ pour le son et $7 \mathrm{~F}$ pour la farine basse de riz de l'Office du Niger.

L'évolution de la production de son et farine basse de riz pour les cinq prochaines années est résumée en annexe.

\section{I.3. Les sous-produits des huileries}

Le principal Centre de production des huiles comestibles est l'huilerie de Koulikoro, située à $60 \mathrm{~km}$ de Bamako, gérée par la S.E.P.O.M. (Société d'Exploitation des Produits Oléagineux du Mali). Elle produit de l'huile d'arachide et de coton, du beurre de karité. Les résidus qui intéressent l'élevage sont les tourteaux d'arachide el de coton, obtenus d'après la méthode d'extraction par pression. Ils conticnnent encore 4-6 p. 100 de matière grasse.

La capacité de l'huilerie est d'environ 30000 tonnes d'arachide coques par an. Le rendement à l'usinage est le suivant :

Coque ............. 30 p. 100, 9000 tonnes

Huile ............. 28 p. 100,8400 tonnes

Tourteau .......... 42 p. 100, 12600 tonnes

La valeur bromatologique du tourteau de Koulikoro est donnée en annexe. Nous n'avons pu donner encore une analyse du tourteau de coton dont la production démarre à peine.

La coque d'arachide est brûlée à l'usine pour faire marcher la chaudière. Les expériences de CALVET et VALENZA à Dakar ont montré l'intérêt de la coque d'arachide mélassée daus l'engraisseinent des bovins.

En 1971, la S.E.P.O.M. a produit 14382,64 tonnes de tourteau d'arachide dont 12000 tonnes ont été exportées pour une valeur de 632 millions de francs maliens.

L'huilerie, qui vient d'être asrandie, va triturer dans les années à venir 65000 tonnes d'arachide coque donnant environ 27000 tonnes de rourteau. Les installations permettront de traiter également 25000 tonnes de graines de coton. On estime que $100 \mathrm{~kg}$ de graines de coton donnent $14-16 \mathrm{~kg}$ d'huile et 84 à $85 \mathrm{~kg}$ de tourteau (3). Le tableau donné en annexe

(3) Marcello Piccioni. Dictionnaire des aliments pour les animaux. résume les prévisions de production de sous-produits des huileries pour les 5 prochaines znnées. Ce tableau ne comporte pas le tourteau de coton dont les caractéristiques ne sont pas encore établies.

\section{I.4. Les graines de coton}

Le coton est produit par la C.F.D.T. (Compagnie Française des Fibres Textiles) dans les régions de Sikasso, Bamako, Ségou et bientôt dans celle de Kayes. L'égrenage du coton grain se fait daus 6 usines dont les productions théoriques en graines de coton sont les suivantes :

\begin{tabular}{|c|c|}
\hline Bamako ..... & 10000 tonnes \\
\hline Ségou. & 5000 tonnes \\
\hline Sikasso $\ldots \ldots \ldots$ & 15000 to \\
\hline Koutiala, 1, 2, 3 & $\begin{array}{r}41000 \\
5000\end{array}$ \\
\hline
\end{tabular}

La production cotonnière a pris un essor remarquable ces dernières années. En 1972-73, la production de graines de coton a atteint environ 25000 tonnes et les prévisions pour les 5 prochaines années sont résumées dans le tableau présenté en annexe.

Les graines de coton sont vendues à $7516 \mathrm{FM}$ la tonne logé carreau usine. La valeur fourragère est de $1,1 \mathrm{UF} / \mathrm{kg}$ et de $105 \mathrm{~g} \mathrm{MAD} / \mathrm{kg}$ (analyse I.E.M. V.T.).

\section{I.5. Les sous-produits de sucrerie}

Les sous-produits issus du broyage des cannes et de la distillerie du jus sont la bagasse et la mélasse.

La bagasse, composée surtout de cellulose, représente 30 à 40 p. 100 du poids de canne broyée. Elle sert de combustible dans l'usine.

La mélasse représente 3,5 à 4,5 p. 100 du poids de la canne broyée.

La production théorique de l'usine de Dougabougou est de 2100 tomnes de mélasse.

En 1971-72 la production s'élevait à 1107 tonnes dont 300 tonnes ont été utilisées dans l'alimentation du bétail, et 1307 tonnes pour la production de l'al cool, dans la fabrique installée près de la sucrerie. On estime qu'il faut une tonne de mélasse pour obtenir 250 litres d'alcool à $90^{\circ}$.

En vitesse de croisière, la sucrerie de Séribala doit broyer 150000 tonnes de canne correspondant à une production théorique de 5250 à 6750 tonnes de mélasse, ce qui portera notre production totale à 7350 8850 tonnes.

La valeur fourragère de la mélasse est de 0,75 UF par kilo et $10 \mathrm{~g}$ de $\mathrm{M} \Lambda \mathrm{D}$ par kilo (analyse I.E.M.V.T.).

\section{I.6. Les sous-produits des abattoirs}

Les sous-produits d'abattoir sont constitués par les farines de viande, de sang et d'os.

Seul l'abattoir frigorifique de Banako d'une capacité théorique de 10000 tonnes de viande par an possède un atelier de fabrication de farines animales. Les viandes saisies, le sang et les os sont transformés en farine de viande, de sang, en poudre d'os. Les quantités actuellement produites sont insuffisantes. L'abattoir n'atteint pas sa capacité d'abattage et il n'existe pas un service d'équarrissage pouvant approvisionner l'atelier en matière première. En 1971, les quantités de farines animales produites ont été les suivantes :

Farine de viande $\ldots \ldots \ldots \ldots \ldots \ldots, 1750 \mathrm{~kg}$

Farine de sang $\ldots \ldots \ldots \ldots \ldots \ldots \ldots \ldots .2350 \mathrm{~kg}$

Poudre d'os ................... $11702 \mathrm{~kg}$

Soit un total de .............. $15802 \mathrm{~kg}$

I.e tonnage des sous-produits d'abattoir est done 
assez faible. Dans les perspectives d'avenir la production qui peut être portée à 45 tonnes ne couvrira même pas les besoins nationaux.

Les prix de cession de ces sous-produits sont les suivants :

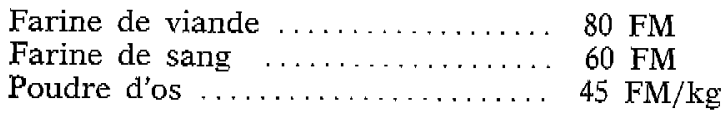

\section{I.7. Les sous-produits de la pêche}

Les sous-produits de la pêche ont deux origines: - la conserverie de poisson de Mopti,

- les déchets de poissons fumés et séchés dans les zones de pêche non encadrées.

\section{I.7.1. La conserverie de Mopti}

L'Opération pêche installée à Mopti a une conserverie de poisson qui produit des filets de « capitaine " fumés, des sardines. Les déchets compreunent les têtes et les arêtes qui sont recupérées et soumis à une cuisson prolongée dans une chaudière permettant de séparer l'huile du résidu qui, séché, donne du granulé qu’on transforme en farine de poisson. drées

1.7.2. Les déchets de poissons des zones non enca-

Le long de la vallée du Niger vivent de nombreux pêcheurs qui drainent vers les centres de consommation des tonnages importants de poissons séches au soleil ou fumés. Les méthodes de traitement et de conditionnement entraînent des déchets importants qui sont vendus au Centre de Recherches Zootechniques de Sotuba, Niono et Centre Avicole). Les quantités pouvant être récupérées pour l'alimentation des anjmaux sont estimées à environ 440 tonnes par an.

L'analyse bromatologique de quelques échantillons est donnée en annexe.

Le poisson séché est vendu à $60 \mathrm{FM} / \mathrm{kg}$ rendu à Bamako.

\section{I.8. Autres sous-produits}

Nous citons ici pour mémoire les drêches de la fabrication artisanale de bière locale (dolo) avec du mil ou du sorgho gerné, les sons de mil de sorgho et de maîs issus des moulins artisanaux ou du pilage de ces céréales pour l'extraction du son. Ces aliments d'une bonne valeur fourragère, n'ont pas fait l'objet d'enquête sérieuse. On peıtt cependant noter qu'ils sont intégralement utilisés pour l'alimentation des moutons de case, des vaches laitières et des volailles. Ils peuvent constituer un appoint fourrager non négligeable dans un programme d'embouche paysanne. Nous donnons dans le tableau en annexe la valeur bromatologique d'un échantillon de son de mil prélevé à Mopti (Coulomb), enquête du troupeau dans l'étude du Développement de l'Elevage en 5 ré gions.

\section{I.9. Bilan général}

Le tableau en annexe récapitule les divers sousproduits et l'évolution prévisible des quantités compte tenu des objectifs de production agricoles. Les teneurs en énergie et en matières azotées digestibles ont été calculées en prenant les valeurs moyennes ci-dessous par kilo.

$\begin{array}{lcc}\text { Aliments } & \mathrm{UF} / \mathrm{kg} & \mathrm{MAD}: \mathrm{g} / \mathrm{kg} \\ \text { Paille de riz } \ldots \ldots \ldots \ldots & 0,30 & 0 \\ \text { Bouts blancs de canne } \ldots & 0,15 & 5 \\ \text { Fanes d'arachide } \ldots \ldots \ldots & 0,40 & 6 \\ \text { Son de riz } \ldots \ldots \ldots \ldots & 0,32 & 31 \\ \text { Farine basse de riz } \ldots \ldots \ldots & 1,10 & 70 \\ \text { Mélasse de canne } \ldots \ldots \ldots & 0,75 & 10 \\ \text { Graines de coton } \ldots \ldots \ldots & 1,10 & 105 \\ \text { Tourteau d'arachide } \ldots \ldots & 0,92 & 280\end{array}$

Farine de viande $\ldots \ldots \ldots . \quad 1,07 \quad 489$

Farine de sang $\ldots \ldots \ldots \ldots, \quad 0,68 \quad 592$

Poudre d'os (porc) ....... $0,40 \quad 262$

Farine de poisson fumé .. $0,81 \quad 592$

Il est évident que la valorisation d'un disponible fourrager aussi important par l'élevage améliorera considérablement nos productions animales. Il s'agit d'une option économique qui doit être fondée sur une analyse approfondie des différentes possibilités d'exploitation de cette richesse nationale. C'est cette question que nous voudrons maintenant aborder en rassemblant ici quelques données économiques relatives à l'utilisation de ces aliments.

\section{II. - DESTINATION ACTUELLE DES SOUS- PRODUITS AGRO-INDUSTRIELS ET ASPECTS TECHNIQUES ET ECONOMIQUES DE LEUR UTILISATION DANS LALIMENTATION DES ANIMAUX}

\section{II.1. Destination actuelle}

Les sous-produits agro-industriels du Mali connaissent trois emplois : exportation, alimentation animale, source de matière première pour certaines industries.

\section{II.1.1. Sous-produits exportés}

Ce sont les graines de coton et les tourteaux d'ara chide et de coton.

Les graines de coton sont généralement exportées par la S.O.M.I.E.X. vers la France et le Japon. Les prix moyens à l'exportation sont les suivants :

\section{F.O.B. Abidjan ............ $27667 \mathrm{FM} /$ tonne \\ F.O.B. Dakar ............ $27860 \mathrm{FM} /$ tonne}

L'huilerie de Koulikoro paie la graine à $13516 \mathrm{FM} /$ tonne tandis qu'elle est cédée à l'élevage à $7516 \mathrm{FM}$ la tonne. Notons que les quantités livrées aux Centres Zootechniques et aux ateliers d'embouche bovine de l'Office du Niger ont été limitées jusqu'iç à quelque 2000 tonnes par an.

Le tourteau d'arachide: Il est exporté vers la Côted'Ivoire, la France, l'Allemagne Fédérale et la Suisse. Le prix à l'exportation varie entre 51000 et $53000 \mathrm{FM}$ / tonne F.O.B. Abidjan. Les prix intérieurs sont $20 \mathrm{FM} /$ $\mathrm{kg}$ pour les Centres Zootechniques et 28 FM pour les autres clients.

II.1.2. Les sous-produits destinés aux industries de transformation

Les graines de colon: Une partie de ce sous-produit est destinée à l'usine de Koulikoro qui va produire du tourteau de graines de coton décortiquées et dont la valeur alimentaire est supérieure à celle de la graine : $1,02 \mathrm{UF} / \mathrm{kg}$ et $373 \mathrm{~g}$ de MAD environ d'après KELLNER.

La mélasse: Elle est utilisée à Dougabougou pour la fabrication d'alcool. La capacité théorique de la fabrique est de 700000 litres, mais la production jusqu'ici tourne autour de 300000 litres en raison des difficultés rencontrées pour le placement de l'alcool à l'extérieur. Actuellement les demandes de la clientèle étrangère ont augmenté. La Pharmacie Populaire exporte l'alcool à $78 \mathrm{~F} \mathrm{CFA}$ le litre (156 FM). La sucre rie de Séribala ne prévoit pas de fabrique d'alcool. Les besoins théoriques de l'usine actuelle $(2800 \mathrm{t}$ ) en mélasse, seront largement couverts par les dewx sucreries laissant encore un disponible au moins égal à 5000 tonnes.

\section{II.1.3. Les autres sous-produits}

Ils sont partiellement utilisés en alimentation des animaux (paitle, fanes d'arachide, bouts blancs de 
canne) ou totalement consommés (issus du riz, farines d'abattoirs).

\section{II.2. Aspect technique de l'utilisation des sous-} produits agro-industriels

L'alimentation rationnelle des animaux a fait largement appel aux sous-produits dans les pays d'Europe et d'Amérique : tourteau d'arachide, de coton, sons des céréales, sous-produits d'abattoirs, etc. Dans la plupart des pays de l'Afrique de l'ouest, Ja composition des provendes pour les volailles, porcs, vaches laitières, comporte des sous-produits quand ils sont disponibles.

L'utilisation massive de ces stocks de fourrages dans les unités modernes de production animale ne fait que démarrer, et cela grâce aux travaux de recherches effectués au cours des dix dernières années. Les travaux se sont intéressés aux aspects les plus divers (valeur bromatologique, composition des rations, consommation par le bétail tropical et efficacité dans l'embouche).

Les chercheurs de l'I.E.M.V.T. (CALVET, VALENZA), ont étudié les rations à base de coques d'arachide mélassées à $15-20$ p. 100 et supplémentées par des sons, des farines de céréales ou de l'urée. Des performances de plus de $1000 \mathrm{~g}$ de grain journalier en 4 mois ont été enregistrées chez les bovins de race Zébu Gobra. Ces mêmes auteurs ont expérimenté d'autres régimes comportant du tourteau d'arachide, des graines de coton, de l'urée, des céréales et des sons. Les gains journaliers obtenus sur N'Dama, Djakoré, Zébu Gobra et Zébu Maure se situent entre $809 \mathrm{~g}$ et $1152 \mathrm{~g}$.

Au Mali, dans le cadre de la préparation du dossier de factibilité pour la création d'une station d'embouche dans la région de Niono, ANDREA FASOLA, en collaboration avec les techniciens de la Station du Sahel a conduit une expérience d'embouche intensive des boufs de 4-5 ans de race peule et maure en utilisant une ration comportant de la paille de riz, du son et de la farine basse de riz, des graines de coton et de l'urée. Des gains de poids de $1100 \mathrm{~g}$ par jour ont été réalisés en 60 jours.

Depuis 1968, nous avons expérimenté à la Station du Sahel et à la sucrerie de Dougabougou, diverses rations à base de paille de riz ou de bouts blancs de canne comme fourrage, grossiers et concentrés, comprenant graines de coton et son, et farine de riz mélassé, urée. En embouche intensive, des gains de poids de plus de $800 \mathrm{~g}$ ont été obterzus en 120 jours. En embouche, sur pâturages naturels supplémentés, de jeunes taurillons de 18 à 24 mois atteignent un croît quotidien moyen de $476 \mathrm{~g}$ sur une période de 12 mois.

Il convient de relever quelques indices de consommation forts intéressants :

Expérience sur Gobra avec coque d'arachide mélassée : 7,04 UF (I.E.M.V.T.) ;

Expérience sur Zébu Maure et Peul avec paille de riz et graines de coton farine basse de rix : 7,21 UF (Station du Sahel et 7,04 UF, FASOLA);

Expérience sur Zébu Peul et Maure avec bouts blancs de canne et graines de coton mélassées : 10,10 UF (Station du Sahel).

Ces résultats techniques dont l'amélioration fait l'objet des travaux dans divers instituts de recherches en Afrique Occidentale ont déjà permis de lancer des ateliers d'engraissement intensif dans certains pays : Sénégal, Mali. Au Mali, l’Office du Niger vient d'installer deux feed-lots à Dougabougou et à Molodo, dont l'alimentation est à base de sous-produits agro-industriels (paille de riz, bouts blancs de canne, farine basse de riz, mélasse et graines de coton). La capacité annuelle des deux ateliers, qui était de 2500 têtes par an sera portée à 6000 têtes au cours de la campagne 1973-74.

On peut donc conclure que l'aspect technique de l'utilisation des sous-produits agro-industriels dans la modernisation de la production de viande bovine dans nos pays ne pose plus des problèmes insurmontables. Même s'il reste quelques questions relatives aux structures de production (embouche industrielle ou embouche paysanne) et à la taille optimale des unités de production, l'on doit reconnaître que les goulots d'étranglement les plus importants sont d'ordre économique.

II.3. Aspect économique de l'utilisation des sousproduits agro-industriels dans les productions animales

Si la transformation en protéines animales des fourrages que nous venons d'énumérer, paraît tech niquement réalisable, il reste à le faire de façon économique. En effet, la modernisation des productions animales doit contribuer au développement économique d'un pays comme le Mali où l'élevage constitue l'une des principales ressources d'exportation. L'insertion de structures modernes de production dans des circuits traditionnels peu organisés ne manque pas de poser des problèmes dont la solution conditionne l'avenir de ces structures de production. Les facteurs économiques qui influeront sur le déve loppement de la nouvelle technique sont : le prix de l'animal maigre adulte ou du jeune animal, le coût des installations et du transport, le prix de la viande.

\section{II.3.1. Le prix de l'animal}

Il connaît au Mali des variations saisonnières importantes. Il est encore trop tôt pour préciser l'évolution de ce prix face au besoin des ateliers d'embouche.

De 1969 à 1973 le prix des aninnaux achetés par l'Office du Niger est passé de :

$12500 \mathrm{FM}$ à $14000 \mathrm{FM}$ pour les jeunes de 18 à 24 mois ;

$22500 \mathrm{FM}$ à $28000 \mathrm{FM}$ pour les adultes de 4 à 5 ans :

$32500 \mathrm{FM}$ à $36000 \mathrm{FM}$ pour les adultes de 6 à 10 ans.

La demande des ateliers étant encore faible, il faut s'attendre à des augmentations dès que le volume du bétail par les centres d'embouche augmentera, sans qu'il soit possible de dire avec suffisamment de précision dans quelles fourchettes se situeront les hausses de prix. Un facteur de stabilisation peut être constitué par la conversion partielle de la qualité du bétail exporté, si les marchands exportateurs donnent leur préférence aux animaux engraissés comme c'est déjà amorcé avec ceux sortis des feed lots de l'Office du Niger.

\section{II.3.2. Les installations}

En embouche industrielle comme paysanne, les installations doivent être simples, fonctionnelles et réalisées avec du matériel local robuste (bois de teck, ronier). Il peut être parfois économique d'utiliser les matériaux de récupération (fers, tôles, etc.).

\section{II.3.3. Le prix de la viande}

Il est l'un des facteurs le plus important. Les prix officiels actuellement appliqués au Mali sont bas, Inême pour la production traditionnelle de viande : (275 FM/kg de viande avec os, $300 \mathrm{FM} / \mathrm{kg}$ sans os). Ils peuvent constituer un frein dans le cadre d'une production moderne de viande où les coûts de production malgré certains facteurs favorables peuvent atteindre facilement la limite de l'irréductible. L'O.M. 
B.E.V.I. étudie actnellement des mesures tendant à résoudre cette question de prix. A titre indicatif nous donnons dans le tableau ci-dessous les coûts de production obtenus avec quelques régimes alimentaires à Dougabougou. Il convient de noter que le prix des graines de coton est très bas. Dans le présent calcul il s'agissait de la production de l'Office du Niger dont le coût rendu à l'atelier était de 8 FM.

Par ailleurs, dans l'étude des avantages à l'exportation ou à la consommation intérieure, le prix de référence est celui de l'exportation.

Le prix de revient du kilo de viande a été le suivant

Lot I : $300 \mathrm{FM}$, poids moyen de carcasse : $168,57 \mathrm{~kg}$; Lot II : $330 \mathrm{FM}$, poids moyen de carcasse : $164,25 \mathrm{~kg}$; Lot III : $290 \mathrm{FM}$, poids moyen de carcasse : $166,80 \mathrm{~kg}$; Lot IV : $315 \mathrm{FM}$, poids moyen de carcasse : $156,60 \mathrm{~kg}$; Lot V : $300 \mathrm{FM}$, poids moyen de carcasse : 168,40 kg.

De meilleurs résultats économiques ont été obtenus par l'Office du Niger au cours de la campagne de production 1972-1973 avec des poids de carcasse compris entre 180 et $235 \mathrm{~kg}$. Il reste cependant évident que le prix de $300 \mathrm{FM} / \mathrm{kg}$ de viande sur pied ne permet pas de commercjaliser cette production au prix officiel actuellement en cours. C'est pourquoi elle est vendue dans les boucheries modernes tenues par la S.O.M.B.E.P.E.C. à un prix plus rémunérateur, ou exportée sur les marchés extérieurs.

A moins d'une modification de la politique des prix de la viande en faveur d'un paiement à la qualité, le marché intérieur ne permet pas de valoriser les animaux engraissés.

Les marchés extérieurs par contre peuvent offrir des perspectives plus intéressantes. En effet les animaux engraissés fort demandés sur les marchés des Ivoiriens et Ghanéens, ont pu être vendus à Abidjan en 1972 à un prix variant entre 45000 et $55000 \mathrm{~F}$ C.F.A. par bovin de 380 à $420 \mathrm{~kg}$. Le bétail traditionnel de type export est vendu à $30000-40000 \mathrm{~F}$ C.F.A. Pour les mêmes frais d'exportation le marchand commercialisant du bétail engraissé réalise un bénéfice moyen de $30000 \mathrm{FM}$ si l'on prend les valeurs moyennes de 70000 FM l'animal type export traditionnel et 100000 FM l'animal engraissé. Bien qu'il ne soit pas prudent de tabler sur le prix dans l'évaluation économique de l'embouche bovine, on peut affirmer que la conjoncture que traversc actuellement l'élevage sahélien et le déficit croissant de la viande dans le monde et en Afrique favoriseront dans les pays d'élevage le développernent de structures susceptibles de mieux valoriser les sous-produits exportés. Une telle évolution conçue dans le cadre d'une stratégie globale de développement de l'élevage peut exercer à long terme une influence décisive sur la transformation et la productivité de l'élevage pastoral sahélien.

Quelques mots maintenant sur les grandes lignes d'une telle stratégie tendant à valoriser au maximum nos ressources fourragères disponibles.

\section{III. - STRATEgIE GENERALE DE L'UTILI- SATION DU DISPONIBLE FOURRAGER EN VUE DE LA MODERNISATION DE NOS PRODUC- TIONS ANIMALES}

La sécheresse exceptionnelle de 1972 vient de rappeler la fragilité de l'élevage pastoral sahélien maintenu grâce à un équilibre instable entre l'animal et son milieu et facilement rompu par l'un des facteurs de variation suivants : augmentation numérique du cheptel, diminution des ressources alimentaires. La plupart des pays sahéliens sont préoccupés par la recherche de voies et moyens permettant de mieux exploiter l'espace pastoral du Sahel. L'utilisation de ressources fourragères venant des industries de transformation constitue un des volets importants des facteurs d'amélioration de cet élevage. Une fois prise la décision de valoriser par les productions animales ce disponible fourrager, Ies grandes lignes d'action sont les suivantes en ce qui concerne les bovins :

1) Exploitation précoce des mâles à sortir du troutpeau à 12-18 mois et leur élevage en embouche dans des structures d'accueil (ranch d'embouche paysanne). En conséquence amélioration des facteurs de multiplication du troupeau sahélien, par décharge des pâturages et augmentation en fonction de la capacité des pâturages des femelles reproductrices;

2) Création de structure moderne en aval de cet élevage sahélien : ranch, atelier d'engraissement intensif, embouche paysanne. L'installation des unités d'embouche industrielle doit se faire en fonction du disponible en bétail mais surtout en fonction de la concentration des ressources alimentaires. Dans cette optique la zone de l'Otfice du Niger offre les conditions jdéales pour le développenent d'une embouche aussi bien industrielle que paysanne. Les ressources fourragères de cette zone sont constituées surtout par des aliments énergétiques, qu'il faut valoriser par l'apport d'aliments protidiques qui peuvent être des sous-produits (graines de coton, tourteau) ou des fourrages cultivés (phaséolus lathyroides ou légumineuses adaptées au milieu);

3) Orientation de nos exportations de bovins sur pied et de viande bovine vers une marchandise do qualité par substitution progressive du bétail engraissé traditionncllement cxporté. Il est encore trop tôt pour dire à quel rythme se fera cette évolution et quel volume de bétail sera concerné aux différentes phases. Les exportations de bovins en 1972, selon les estimations du Service de l'élevage, se sont situées entre 130000 et 150000 têtes. Il est certain que ce chiffre doit diminuer dans les années à venir en raison des effets de la sécheresse. En retenant le chiffre moyen de 140000 et en lui appliquant une réduction de 60 p. 100 , nos exportations ne porteraient plus que sur 56000 têtes environ. En admettant un poids moyen de carcasse de $120 \mathrm{~kg}$ par zébu, les 56000 bovins correspondent à 6720 tomnes de viande. Par l'engraissement donnant des carcasses de $180 \mathrm{~kg}$, le tonnage de viande pour le mêrne effectif serait dé 10080 tonnes. La production de ces animaux nécessiterait environ 17000 tonnes de graines de coton.

Une telle évolution ne peut se faire qu'avec le concours des professionnels du bétail et de Ja viande.

C'est pourquoi les pouvoirs publics maliens attachent une importance particulière à l'insertion de cette couche sociale dans les structures modernes de production et de commercialisation de bovins et viande bovine, par l'introduction du crédit dans la production animale:

4) Développement de l'embouche paysanne dans les zones de développement agricole fortement encadrées (zone opération coton, opération arachide, opération riz, Office du Niger);

5) Développement des autres productions animales : volailles, porcs, productions laitières par l'utilisation des sous-produits d'abattoir, de pêche et du tourteau d'arachide. La création d'une usine d'aliments du bétail pouvant fabriquer des aliments concentrés azotés ou des aliments complets constituc dans ce cadre une nécessité. Il est certain que le ravitaillement en céréales peut poser des difficultés à la réalisation d’un tel projet. Mais conçu dans le cadre d'une évolution globale du secteur de production animale, la priorité qu'il requiert doit orienter les productions céréalières vers la satisfaction de ce besoin. Si on recherche les productions animales sus- 
ceptibles d'être réalisées par une politique tendant à une consommation intégrale de nos sous-produits, on constate que le déficit se situe au niveau de l'apport énergétique pour les porcs et volailles et des protides pour les bovins.

Il s'agit bien sûr d'un schéma théorique dont la réalisation pose de nombreux problèmes socio-économiques. L'exportation des sous-produits rapporte actuellement plus d'un milliard de francs maliens par an. La reconversion de cette valorisation traditionnelle est conditionnée à des mutations profondes de nos méthodes d'élevage et d'exportation du bétail. Tous les grands projets du développement de l'élevage élaborés ou en cours d'élaboration au Mali sont conçus dans l'optique de cette nouvelle stratégie et leur réalisation doit aboutir à l'amélioration de la productivité de notre élevage.

\section{CONCLUSION}

1) L'existence de nombreux sous-produits agroindustriels au Mali et les perspectives de leur augmentation constituent des facteurs favorables à une amélioration des productions animales. Leur localisation géographique et leur coût relativement bas en rendent la transformation en protéiques animales assez rentable pour le pays.

2) Les recherches effectuées ces dernières annces par l'Institut d'Elevage et de Médecine Vétérinaire des Pays Tropicaux au Sénégal, au Cameroun, en
Côte-d'Ivoire et par l'Institut d'Economie Rurale au Mali ont montré que le bétail tropical pouvait répondre favorablement aux techniques rationnelles d'alimentation.

La poursuite de certaines recherches de base (digestibilité des fourrages tropicaux, physiologie et métabolisme chez nos zébus) doit permettre d'élargir encore le front de la valorisation de stocks considérables d'aliments jusqu'ici gaspillés ou mal exploités.

3) La nécessité pour les pays sahéliens de grand élevage d'accroître leur production de viande malgré la catastrophe causée au cheptel par la sćcheresse exceptionnelle de 1972, leur impose d'élaborer rapidement une stratégie du développement de l'élevage dans laquelle les structures modernes de production appuyés sur l'existence des sous-produits agro-industriels, trouveront leur insertion. La valorisation maximale de ces aliments représente dans cette stratégic une première étape, la production fourragère intensive devant constituer la deuxième étape pour garantir le développement d'un élevage à plus grande productivité.

4) Ces mutations souhaitables dans les pays sahéliens seront les résultats des efforts des techniciens, économistes, des institutions de financement. Elles seront surtout le plus beaty fruit d'une coopération régionale dans tous les domaines et à tous les niveaux entre pays de la côte et pays de l'intérieur. Et c'est sur l'espoir que ces efforts soient entrepris de part et d'autre et nous permettent de contribuer plus efficacement à la lutte contre la faim protéinique et le sous-développement que je voudrais conclure cet exposé.

Coût de la production ajoutée.

\begin{tabular}{|c|c|c|c|c|c|c|c|c|c|c|}
\hline \multirow[t]{2}{*}{ Poste des dépenses } & \multicolumn{2}{|c|}{$\begin{array}{l}\text { Régime I } \\
\text { Gain de poids } \\
3.885 \mathrm{~kg}\end{array}$} & \multicolumn{2}{|c|}{ 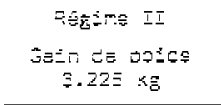 } & \multicolumn{2}{|c|}{ 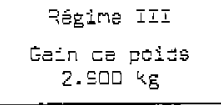 } & \multicolumn{2}{|c|}{$\begin{array}{l}\text { Régime IV } \\
\text { Gein de Poids } \\
3.060 \mathrm{~kg}\end{array}$} & \multicolumn{2}{|c|}{$\begin{array}{l}\text { Rógime } V \\
\text { Eạin de poids } \\
3.105 \mathrm{~kg}\end{array}$} \\
\hline & Total & par kg & $T=2 \pm 1$ & $p=545$ & Tøะล? & par kg & Total & pár k站 & Total & par $\mathrm{kg}$ \\
\hline Amortissement & 29.430 & 7,58 & $30 . \equiv 20$ & 9,49 & 27.250 & 5,40 & 29.430 & 9,62 & 28.340 & 9,12 \\
\hline Alimentatian & 315.920 & 82.35 & 419.615 & 930.05 & 206.255 & $7 i, 12$ & 287.390 & 93,92 & 341.194 & 109,89 \\
\hline Personnel & 21.365 & 5,49 & 130.050 & 40,32 & 120.725 & 41,42 & 129.735 & 42,40 & 116.870 & 37,64 \\
\hline Frais véterinaires et divers & 17.712 & 4.55 & 70.564 & 5,35 & $: 7.550$ & 5,05 & 16.354 & 亏, 19 & 17.056 & 5,49 \\
\hline Pertes & 41.796 & 10,75 & 50.400 & $1 \equiv, 53$ & 31.025 & 27.53 & 37.231 & 12,17 & 19.703 & 6,35 \\
\hline Frais génëraux $10 \%$ & 53.012 & 13,56 & 54.937 & 20,14 & 45.220 & 15,55 & 50.274 & 16,42 & 52.316 & 16,85 \\
\hline Totaux & 483.235 & 124,38 & 714.316 & 221,45 & 497.424 & 177.51 & $553 . \square 14$ & 180,72 & 575.479 & 185,34 \\
\hline
\end{tabular}


Evaluation des sous-produits agro-industriels disponibles et leurs valeurs nutritives et évolution (milliers d'unités fourragères et tonnes de M.A.D.).

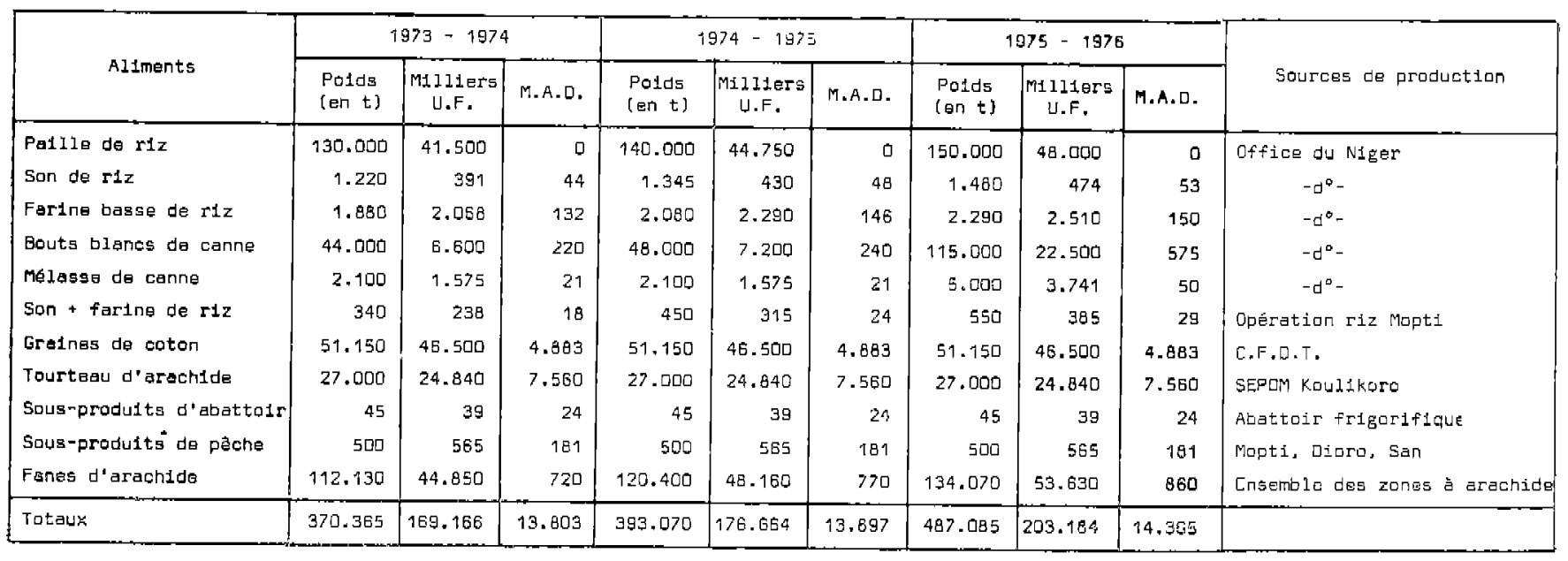

\begin{tabular}{|c|c|c|c|c|c|c|c|}
\hline \multirow{2}{*}{ Alím四亡E } & \multicolumn{3}{|c|}{$1976-1977$} & \multicolumn{3}{|c|}{$1977-1978$} & \multirow[b]{2}{*}{ Sources de production } \\
\hline & $\begin{array}{l}\text { Poids } \\
\text { [en t] }\end{array}$ & $\mid \begin{array}{c}\text { Milliers } \\
\text { U.F. }\end{array}$ & M.A.D. & $\begin{array}{l}\text { Poids } \\
{\left[\begin{array}{ll}\text { en } t]\end{array}\right.}\end{array}$ & $\begin{array}{c}\text { Miliners } \\
\text { U.F. }\end{array}$ & M.A.D. & \\
\hline Paille de riz & 180.000 & 51.200 & $\square$ & 08.000 & 57.500 & 0 & affice du Niger \\
\hline San de riz & 1.610 & 515 & 59 & 1.770 & $56 \mathrm{E}$ & 63 & $-d^{\mathrm{D}}-$ \\
\hline Farine basse de riz & 2.485 & 2.740 & 174 & 2.730 & 3.000 & 191 & $-]^{0}-$ \\
\hline Eouts blanes de canne & 115.000 & 22.500 & 575 & 115.000 & 22.500 & 575 & $-0^{\circ}-$ \\
\hline Mélasse de еапле & 5.000 & 3.741 & 50 & 5.000 & 3.741 & 50 & $-0^{\circ}-$ \\
\hline Son + farine de riz & 665 & 465 & 37 & 760 & 532 & 40 & Dpśratian riz Mopti \\
\hline Gruimes de coton & 51.150 & 46.500 & 4.893 & 51.150 & 46.500 & 4.883 & [.F.D.IT. \\
\hline Tourteau d"arachide & 27.000 & 24.840 & 7.560 & 27.000 & 24.840 & 7.550 & SEPOM Koulikoro \\
\hline Sous-prodults d'abattoir & 45 & 39 & 24 & 45 & 39 & 24 & nbattoir frigarifique \\
\hline Sous-produfts de péche & 500 & 565 & 181 & 500 & 565 & 181 & Mopti, Jioro, San \\
\hline Fanes d'srachide & 147.880 & 59.150 & 950 & 161.200 & 54.480 & 1.030 & Ensemble des zones à arachide \\
\hline Tataux & 511.335 & 212,255 & 14.493 & 445.155 & 224.2 追3 & 14.597 & \\
\hline
\end{tabular}

Avec le déficit pluviométrique, les prévisions nc seront certainement pas réalisécs. En particulier le stock de graines de coton disponible, après prélevement des semences, ne dépassera pas 15000 tomes.

Sous-produits disponibles pour embouche bovine.

\begin{tabular}{|c|c|c|c|c|c|c|c|c|c|c|}
\hline \multirow{2}{*}{ Aliments } & \multicolumn{2}{|c|}{$1973-1974$} & \multicolumn{2}{|c|}{$1974-1975$} & \multicolumn{2}{|c|}{$1975-1975$} & \multicolumn{2}{|c|}{$1976-1977$} & \multicolumn{2}{|c|}{$1977-197 y$} \\
\hline & $\begin{array}{l}\text { Milliers } \\
\text { d'U.F. }\end{array}$ & $\begin{array}{l}\text { Tonnes } \\
\text { de } \\
\text { M.A.D. }\end{array}$ & $\begin{array}{c}\text { Miduiers } \\
\text { du.t. }\end{array}$ & $\begin{array}{c}\text { Tonnes } \\
\text { de } \\
\text { M.A.D. }\end{array}$ & $\begin{array}{c}\text { Milliers } \\
\text { d'ப.F. }\end{array}$ & $\begin{array}{c}\text { Tonnes } \\
\text { de } \\
\text { M.A.E. }\end{array}$ & $\begin{array}{c}\text { Milliers } \\
\text { d'U.F. }\end{array}$ & $\begin{array}{l}\text { Tonnes } \\
\text { de } \\
\text { M.A.D. }\end{array}$ & $\begin{array}{c}\text { Mizliers } \\
\text { d'U.F. }\end{array}$ & $\begin{array}{c}\text { Tonnes } \\
\text { de } \\
\text { M.A.D. }\end{array}$ \\
\hline Paille de riz & 41.500 & 0 & 44.750 & $\square$ & 48.000 & 0 & 57.200 & c & 57.500 & $\square$ \\
\hline Son de riz & 391 & 44 & 430 & 48 & $47 n$ & 53 & 515 & 59 & 566 & E3 \\
\hline Bauts blancs de салпе & 6.600 & 220 & 7.200 & 240 & 22.500 & 575 & 22.500 & 575 & 22.500 & 575 \\
\hline Fanes d'arachica & 44.850 & 720 & 48.160 & 770 & 53.630 & 350 & 59.150 & 950 & 64.480 & 1.030 \\
\hline Mélasse dg canne & 1.575 & 21 & 1.575 & 21 & 3.741 & 50 & 3.741 & 50 & 3.741 & 50 \\
\hline Graines de coton & 46.500 & 4.883 & 46.500 & 4.883 & 46.500 & 4.883 & 45.500 & 4.863 & 46.500 & 4.883 \\
\hline Totaux & 141.416 & 5.889 & 118.815 & 5.962 & 174.845 & 6.421 & 183.805 & 8.517 & 195.287 & E.601 \\
\hline
\end{tabular}


4

Production de viande estimée.

\begin{tabular}{|c|c|c|c|c|c|}
\hline Earacteristiques & $1973-1974$ & $1974-1975$ & $1975-1975$ & $1976-1977$ & $1977-1978$ \\
\hline Nambre d'U.B.T, engraissês & 132.800 & 139.330 & 163.300 & 171.140 & 181.760 \\
\hline Poids vif [l] & 47.800 & 50150 & 58.790 & 61.610 & 65.435 \\
\hline Poids de viande [t] & 25.800 & 27.900 & 31.700 & 33.300 & 35.300 \\
\hline M.A.D. nÉcessaire (t) & 8.755 & 9.195 & 10.778 & 11.295 & 11.995 \\
\hline Defficit en M.A.D. [t] & 2.377 & 3.233 & 4.357 & 4.779 & 5.394 \\
\hline
\end{tabular}

Hypothèses : + 10 U.F. et $600 \mathrm{~g}$ de M.A.D. par $\mathrm{kg}$ de gain de poids vif

+ bovins de $250 \mathrm{~kg}$ gagnant $100 \mathrm{~kg}$ de poids en 140 jours

+ rendement $=54$ p. 100.

5

Valeur bromatologique de quelques échantillons.

\begin{tabular}{|c|c|c|c|c|c|c|c|c|c|c|}
\hline \multirow{2}{*}{ Analyse Prélévements } & \multicolumn{2}{|c|}{ Pailie de riz } & \multicolumn{2}{|c|}{$\begin{array}{c}\text { Eouts blanos } \\
\text { de canne }\end{array}$} & \multirow{2}{*}{$\begin{array}{c}\text { Tourbeal } \\
\text { d'arachida } \\
\text { TEMVT }\end{array}$} & \multicolumn{2}{|c|}{ Fanes d'arachide } & \multirow{2}{*}{$\begin{array}{c}\text { Farine } \\
\text { de poissons } \\
\text { SEgut }\end{array}$} & \multirow{2}{*}{$\begin{array}{c}\text { Son de mil } \\
\text { IEMVT }\end{array}$} & \multirow{2}{*}{$\begin{array}{l}\text { Tourteau } \\
\text { de coton } \\
\text { IENNT }\end{array}$} \\
\hline & 1 it & 294 & 344 & 325 & & IEMVT & SLTUEA & & & \\
\hline Humidité (p.100 de Ja mál.brute) & 5,00 & 3,50 & 87,90 & 68,60 & 6,30 & 12.50 & 4.44 & - & b. 25 & - \\
\hline Matière sèche . . . . . . & 95,00 & 96,50 & 13,10 & 31,40 & $93,7 \square$ & 87,50 & 95,56 & 92,60 & 91.75 & 92,60 \\
\hline Matières protëiques brutes . . & 2,33 & 1.83 & 5.43 & 5,74 & 34,95 & 12,90 & 11,96 & 66.76 & 12.88 & 21,50 \\
\hline Cellulose , . . . . . . & 36,30 & 35,95 & 39,75 & 32,50 & 10,20 & 23,30 & 23,84 & 1,40 & 4.55 & 10,70 \\
\hline Matiere grassa,$\cdot$. . . . . & 1.05 & 1,07 & 1.58 & 0,55 & 8,24 & $1, \bar{B}]$ & 3,06 & 13,89 & 8,47 & 7,70 \\
\hline Mattères minérales totales $\cdot$ ' & 15,98 & 12,27 & 8,35 & 7,17 & 7,68 & 11,20 & 7.80 & 15,14 & 7,15 & - \\
\hline Extractif non azotét. . . . . & 39,33 & 8,83 & 49,69 & 53,64 & 32,63 & 51,00 & 53,35 & - & 58,70 & 26,50 \\
\hline Insaluble chlorhydrique,$\cdot$. & 11,71 & 0,24 & 4,19 & 3,70 & 3,20 & 2,10 & 7,08 & 1,22 & 3.92 & - \\
\hline Calcium . . . . . . . . & 0,293 & 0,22 & 0,29 & 0,21 & $\square, 126$ & 1,86 & 1,10 & 3,86 & 0,13 & 0,21 \\
\hline Phosphore........ & 0,072 & 0,048 & 0,178 & 0,120 & 0.349 & 0,113 & $\square, 23$ & 2.26 & $\square, a 58$ & 1,10 \\
\hline Potessitum . . . . . . . . & 1,98 & 2,24 & 1,87 & 1,70 & 1,08 & 1.62 & - & 1,12 & 0,80 & - \\
\hline Magnósium , . . . . . . . . & 0,117 & 0.10 & 0,14 & 0,19 & 0,328 & 0,62 & - & 0.13 & 0,23 & - \\
\hline Sodium . . . . . . . . & - & - & - & - & - & $\square, 009$ & $=$ & - & - & - \\
\hline 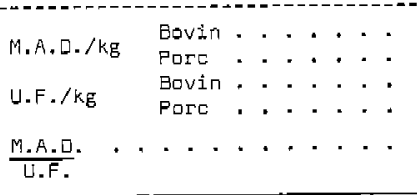 & $\begin{array}{c}- \\
- \\
0,35 \\
-\end{array}$ & $\begin{array}{l}- \\
- \\
0, \triangle 4 \\
-\end{array}$ & $\begin{array}{c}0.10 \\
- \\
5 \\
0\end{array}$ & $\begin{array}{l}0.21 \\
11^{-} \\
52\end{array}$ & $\begin{array}{c}280 \\
0.92 \\
315\end{array}$ & $\begin{array}{l}0.8 \\
- \\
0,80\end{array}$ & 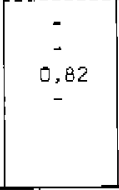 & $\begin{array}{l}572 \\
592 \\
8,7 \\
5,5\end{array}$ & $\begin{array}{c}- \\
- \\
0,95 \\
-\end{array}$ & $\begin{array}{l}340 \\
353 \\
0,99 \\
0,90\end{array}$ \\
\hline
\end{tabular}

